\title{
Tumor-Infiltrating Lymphocytes in Primary Tumor and Bone Marrow in Patients with Gastric Cancer and Overweight
}

Larissa Nikitichna Bubnovskaya ${ }^{1}$, Viktor Michailovich Mikhailenko ${ }^{1}$, Sergey Pavlovich Merentsev ${ }^{2}$ and Dmitriy Sergeevich Osinsky²

1. R. E. Kavetsky Institute of Experimental Pathology, Oncology and Radiobiology, National Academy of Sciences of Ukraine, Vasilkovskaya Str. 45, Kiev-03022, Ukraine

2. City Clinical Oncological Center, Verchovynna Str. 69, Kiev-03115, Ukraine

\begin{abstract}
Immune infiltration in human tumors is a key prognostic factor. In this aspect it has to be noted that the publications concerning the tumor-infiltrating lymphocytes (TILs) under overweight and obesity are limited, especially in the clinical setting in particular concerning gastric cancer. This study has shown that density of TILs consistently associated with body mass index (BMI) and density of cancer-associated adipocytes (CAA) in tumors, decreased significantly in patients with BMI > 30 having high density of CAA. Hypoxia in tumor does not affect this process. Patients with BMI $>30$ having high density of CAA with slight infiltrating of TILs in tumors demonstrated better overall survival than the same patients with BMI $<25$ whereas the same patients with BMI $<25$ but with low density of CAA have shown much longer overall survival. Presence of TILs in BM was not affected by both the high density of CAA and level of hypoxia in primary tumor; moreover it has not been associated with BMI as well as patients survival time. Understanding the metabolic changes that occur in obese individuals may help to pave the way for more effective treatments for patients with gastric cancer having overweight.
\end{abstract}

Key words: $\mathrm{CD}^{+}, \mathrm{CD} 45 \mathrm{RO}^{+}-\mathrm{T}$ lymphocytes, $\mathrm{CAA}$, bone marrow, hypoxia, BMI.

\section{Introduction}

Obesity is a wide-spread condition directly or indirectly connected with an increase in the prevalence of a variety of human diseases. Excess body weight and weight gain have been reported to independently increase the risk of several cancers. Obesity has been linked to more aggressive characteristics of the major common cancers, including breast and prostate cancer, colorectal, endometrial, esophageal, pancreatic, renal cell cancer and thyroid cancer [1]. Most of the available studies have observed modest obesity-associated increases in the risk of non-Hodgkin's lymphoma, multiple myeloma, and leukaemia [2]. However, the risk of

Corresponding author: Bubnovskaya Larissa, Ph.D., senior researcher, research fields: biochemistry, oncology. developing other cancers (melanoma, rectal, and ovarian) is not altered by body mass. In contrast to the convincing evidence that obesity (measured by body mass index, BMI) increases the risk of many different types of cancer, there is an ambiguity in the role of obesity in survival among cancer patients. Some studies suggested that higher BMI decreased mortality risk in cancer patients, a phenomenon called the obesity paradox [3].

Many tumor types (gastric, breast, colon, renal, and ovarian) grow in the anatomical vicinity of adipose tissue and adipose tissue appears to contribute to paracrine interactions in the tumor microenvironment. Solid tumor growth and metastasis require the interaction of tumor cells with the surrounding tissue leading to a view of tumors as tissue-level phenomena rather than exclusively cell-intrinsic anomalies. Due to 
the ubiquitous nature of adipose tissue, many types of solid tumors grow in proximate or direct contact with adipocytes and adipose-associated stromal and vascular components such as fibroblasts and other connective tissue cells, stem and progenitor cells, endothelial cells, innate and adaptive immune cells, and extracellular signaling and matrix components. Adipocytes modulate the tumor microenvironment by promoting angiogenesis, affecting immune cells and altering metabolism to support growth and survival of metastatic cancer cells [4] and understanding the mechanisms of metabolic symbiosis between cancer cells and adipocytes, which should reveal new therapeutic possibilities.

As many of these changes also resemble shifts observed within the tumor microenvironment, proximity to adipose tissue may present a hospitable environment to developing tumors, providing a critical link between adiposity and tumorigenesis [5].

The special place in tumor microenvironment is occupied by the intratumoral hypoxia that influences cancer cell function, alters the extracellular matrix, modulates the tumor metabolic adaptive and immune response, increases angiogenesis, proliferation and metastasis [6]. During interaction with cancer cells under hypoxic condition in tumor microenvironment, adipocytes dedifferentiate into pre-adipocytes or are reprogrammed into cancer-associated adipocytes (CAA). Adipokines secreted from adipocytes likely form a key component of the paracrine signaling in the tumor microenvironment and together with the abundant availability of lipids from adipocytes promote adhesion, migration, and invasion of tumor cells and support tumor progression and uncontrolled growth [7].

Tumor-infiltrating lymphocytes (TILs) are often found in tumors presumably reflecting an immune response against the tumor and are considered one of the hallmarks of cancer development. The classic view of the role of immune cells in cancer has been supplanted by a more complex view of leukocytes having both pro- and anti-tumor properties [8]. In many types of cancer massive infiltration of TILs that are crucial components of antitumor immunity, tends to have a better prognosis, as it was shown for oesophageal squamous cell carcinoma [9], breast cancer [10, 11], head and neck cancer [12], colorectal cancer [13]. Bachmayr-Heyda et al. [14] have confirmed with ovarian cancer patients a significant survival advantage associated with TILs. It was shown

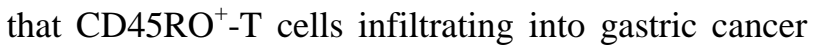
tissues are functional and have significant prognostic value in human gastric cancer [15]. Moreover the presence of CD8- and CD45RO-positive $\mathrm{T}$ cells in bone marrow (BM) of gastric cancer patients demonstrated better overall survival than those with the absence of CD8- and CD45RO-positive T cells in BM [16]. At the same time as it was noted by Mei et al. [17], the prognostic value of TILs in gastric cancer remains controversial because of the limited number of studies.

Conversely, tumors with marked infiltration of TILs tend to have a worse prognosis $[18,19]$.

Thus, both the quantity and quality of TILs are crucial determinants of the outcome of antitumor immune response and it would be paid attention to the suggestion of Pages et al. [20] that immune infiltration in human tumors is a key prognostic factor that should not be ignored.

However, the exact magnitude of TILs on prognosis remains somewhat mysterious due to methodological factors. Improving study quality is an essential step toward uncovering the real clinical relevance of TILs. Gooden et al. [21] have marked that any future studies should be carefully designed, to prevent overestimating the effect of TILs on prognosis.

In this aspect it has to be noted that the publications concerning the TILs infiltration under overweight and obesity are limited, especially in the clinical setting.

Therefore this study was aimed to determine the relative density of $\mathrm{T}$ lymphocytes, $\mathrm{CD}^{+}-$and $\mathrm{CD}_{45 \mathrm{RO}^{+}}$-cells in tumor and $\mathrm{BM}$, to evaluate the 
association between the presence of $\mathrm{T}$ lymphocytes both in tumor and BM, CAA density, tumor hypoxia and their impact on disease outcome in patients with gastric cancer according to the BMI.

\section{Patients and Methods}

\subsection{Patients}

A total of 94 patients ( 62 men and 32 women) with primary gastric cancer were diagnosed and treated at the City Clinical Oncological Center (Kiev). No patient received any pre-operative anti-cancer therapy. Tumors were classified and staged according to the 2002 version of the Union for International Cancer Control (UICC) staging system [22]. Histological types of tumors were evaluated by World Health Organization (WHO) histological classification (2000) [23]. Tissue samples were taken immediately after tumor excision. Preoperatively, 2.0-3.0 mL of BM aspirates from the sternum with conventional cautions to avoid the hit of skin epithelial cells into the sample were obtained.

All patients were thoroughly informed about the study that was approved by the local ethics committee.

\subsection{Immunohistochemical Examination of Tumor Tissue}

Expressions of CD8 and CD45RO as well as Perilipin $\left(\mathrm{Plin}^{+}\right)$as a marker for viable adipocytes were evaluated on deparaffinized slides by means of immunohistochemical staining using specific monoclonal mouse antibodies: clone C8/144B (Dako Cytomation, Denmark), clone UCHL1 (Sigma, USA), and monoclonal rabid antibodies (Perilipin-5/OXPAT Antibody, Termoscientific, USA), respectively. Immunoreactions were detected and visualized with the polymer-peroxidase method (EnVision+/HRP and 3,3-diaminobenzidine; Dako Cytomation, Denmark) followed by counterstaining with Mayer hematoxylin. Negative controls were employed in which the primary antibody was replaced by phosphate-buffered solution (PBS). Intratumoral CD- and Plin5-positive cells were counted per 1,000 cells in each slide and the number of positive cells was reported as a percent.

\subsection{Immunocytochemistry Examination of BM}

For the detection of CD8 and CD45RO T cells in $\mathrm{BM}$ cytospin preparations were fixed by formol-acetone solution ( $\mathrm{pH}$ 6.6). Slides were treated by $0.3 \%$ Triton X-100 solution, followed by incubation in $3 \%$ bovine serum albumin. Cytospins were incubated with primary monoclonal mouse antibodies against CD8 (clone C8/144B, ready-to-use, Dako, Denmark) or with primary monoclonal mouse antibodies against CD45RO (clone UCHL1, Sigma, США) in optimal dilution (1:400); slides were processed with PolyVue HRP Detection System Components (Diagnostic BioSystems, USA). Visualization of peroxidase activity was provided by staining with DAB (Dako, Denmark). Cytospins were counterstained by solution of methyl green (ready-to-use, Dako, Denmark). Cytospins were examined by light microscopy $(\times 1,000)$ to detect CD8- and CD45RO-positive T cells.

\subsection{1 ${ }^{31} \mathrm{P}$ NMR Spectroscopy}

Level of tumor hypoxia was assessed with ${ }^{31} \mathrm{P}$ NMR spectroscopy. ${ }^{31} \mathrm{P}$ NMR spectra of perchloric acid (PCA) tumor extracts were acquired by means of a high-resolution Bruker $400 \mathrm{MHz}$ spectrometer (Widebore Ultrashield, AV-400 Electronics, Germany) using a probe of $5 \mathrm{~mm}$ inner diameter [24].

Level of tumor hypoxia assessed by NMR spectroscopy was ranged following to the metabolic ratio $\mathrm{PME} / \mathrm{Pi}$ value: if $\mathrm{PME} / \mathrm{Pi}<1.0$, hypoxia was appointed as severe; 1.0 $<\mathrm{PME} / \mathrm{Pi}<1.4$-moderate; $1.4<\mathrm{PME} / \mathrm{Pi}<2.0$ - mild and PME/Pi $>2.0$-weak (satisfactory oxygenated) [25].

\subsubsection{BMI $\left(\mathrm{kg} / \mathrm{m}^{2}\right)$}

Patients were classified according to BMI, following the WHO definitions, as underweight, normal $\left(18.5-<25.0 \mathrm{~kg} / \mathrm{m}^{2}\right)$, overweight $(25.0-<30.0$ $\left.\mathrm{kg} / \mathrm{m}^{2}\right)$ or grade 1 obesity $\left(30.0-<35.0 \mathrm{~kg} / \mathrm{m}^{2}\right)$. Patients without unknown BMI and underweight were 
excluded. BMI is commonly used to identify obesity.

\subsubsection{Statistical Analysis}

All statistical analyses were conducted using the NCSS 2000/PASS 2000 and Prism, version 4.03 software packages. The survival proportion was estimated by using the Kaplan-Maier method and differences in survival were analyzed with the log-rank test. Prognostic values of relevant variables were analyzed by means of the Cox proportional hazards model using odds ratio (OR) and $\chi^{2}$ test. Two-tailed $p$ values $<0.05$ were considered statistically significant.

\section{Results}

\subsection{CAA in Tumors of Patients according to BMI}

Individual patient data from a total 94 histological confirmed gastric cancer patients were included in this study (Table 1). The median age was 61.3 years. Median number of CAA in tumors was 26.5\%. We defined this number as the cut-off value and classified all cases into high- or low-density groups. Overall, $48.4 \%$ of tumors were characterized by a low density of CAA and $51.6 \%$ by a high CAA during follow-up Table 2.

Table 1 Patient and tumor characteristics.

\begin{tabular}{|c|c|}
\hline Characteristics & Number, 94, (\%) \\
\hline \multicolumn{2}{|l|}{ Gender } \\
\hline Male & $62(66.0)$ \\
\hline Female & $32(34.0)$ \\
\hline \multicolumn{2}{|l|}{ Age (years) } \\
\hline Median & 61.3 \\
\hline Range & $26-84$ \\
\hline \multicolumn{2}{|l|}{ Tumor location } \\
\hline Upper third & $12(12.8)$ \\
\hline Middle third & $26(27.7)$ \\
\hline Lower third & $53(56.4)$ \\
\hline Total & $3(3.2)$ \\
\hline \multicolumn{2}{|l|}{ UICC stage } \\
\hline I & $23(24.5)$ \\
\hline II & $22(23.4)$ \\
\hline III & 30 (31.9) \\
\hline IV & $19(20.2)$ \\
\hline \multicolumn{2}{|l|}{ Histological type } \\
\hline Adenocarcinoma & 69(73.4) \\
\hline Mucinous adenocarcinoma & $10(10.6)$ \\
\hline Signet-ring cell carcinoma & $12(12.8)$ \\
\hline Undifferentiated carcinoma & $3(3.2)$ \\
\hline \multicolumn{2}{|l|}{ Grade (G) } \\
\hline 1 & $5(5.3)$ \\
\hline 2 & $16(17.0)$ \\
\hline 3 & $66(70.2)$ \\
\hline 4 & $7(7.5)$ \\
\hline \multicolumn{2}{|l|}{ T-classification } \\
\hline $\mathrm{T}_{1}$ & $10(11.6)$ \\
\hline $\mathrm{T}_{2}$ & $21(22.3)$ \\
\hline $\mathrm{T}_{3}$ & 37 (39.3) \\
\hline $\mathrm{T}_{4}$ & $26(27.6)$ \\
\hline \multicolumn{2}{|l|}{ Nodal involvement } \\
\hline $\mathrm{N}_{0}$ & $52(55.3)$ \\
\hline $\mathrm{N}_{1+2}$ & $42(44.7)$ \\
\hline \multicolumn{2}{|l|}{ Distant metastasis } \\
\hline $\mathrm{M}_{0}$ & 83(88.3) \\
\hline $\mathrm{M}_{1}$ & $11(11.7)$ \\
\hline
\end{tabular}


Table 2 Patients with CAAs in tumor in GC by clinical variables.

\begin{tabular}{|c|c|c|}
\hline \multirow[b]{2}{*}{ Variables } & \multicolumn{2}{|c|}{ Patients with CAAs in tumor } \\
\hline & $\begin{array}{l}\text { Low density }(<\text { median }) \\
(N=47,50 \%)\end{array}$ & $\begin{array}{l}\text { High density (> median) } \\
(N=47,50 \%)\end{array}$ \\
\hline \multicolumn{3}{|l|}{ Gender } \\
\hline Male $(N=62)$ & $32(34.0 \%)$ & $30(31.9 \%)$ \\
\hline Female $(N=32)$ & $16(17 \%)$ & $16(17 \%)$ \\
\hline Age (years) median (range) & $61.0(26-84)$ & $61.4(39-81)$ \\
\hline \multicolumn{3}{|l|}{ Histological type } \\
\hline Adenocarcinoma & 36 (76.5\%) & $36(76.5 \%)$ \\
\hline Mucinous adenocarcinoma & $6.0(12.8 \%)$ & $4.0(8.5 \%)$ \\
\hline Signet-ring cell carcinoma & $5.0(10.6 \%)$ & $7.0(14.9 \%)$ \\
\hline Undifferentiated carcinoma & 0 & 0 \\
\hline \multicolumn{3}{|l|}{ Tumor location } \\
\hline Upper third & $4.0(8.5 \%)$ & $8.0(17 \%)$ \\
\hline Middle third & $13(27.6 \%)$ & $12(25.5 \%)$ \\
\hline Lower third & $28(59.6 \%)$ & $25(53.2 \%)$ \\
\hline Total & $2.0(4.2 \%)$ & $2.0(4.2 \%)$ \\
\hline \multicolumn{3}{|l|}{ Grade (G) } \\
\hline 1 & $3.0(6.4 \%)$ & $2.0(4.2 \%)$ \\
\hline 2 & $9.0(19.1 \%)$ & $7.0(14.9 \%)$ \\
\hline 3 & $32(68 \%)$ & 34 (72.3\%) \\
\hline 4 & $3.0(6.4 \%)$ & $4.0(8.5 \%)$ \\
\hline \multicolumn{3}{|l|}{ UICC stage } \\
\hline I & $19(40.4 \%)$ & $4.0(8.5 \%)$ \\
\hline II & $13(27.7 \%)$ & $9.0(19.1 \%)$ \\
\hline III & $10(21.3 \%)$ & $20(42.6 \%)$ \\
\hline IV & $5.0(10.6 \%)$ & $14(29.8 \%)$ \\
\hline \multicolumn{3}{|l|}{ T-classification } \\
\hline $\mathrm{T}_{1}$ & 7 (14.9\%) & $3(6.4 \%)$ \\
\hline $\mathrm{T}_{2}$ & $15(31.9 \%)$ & $6.0(12.8 \%)$ \\
\hline $\mathrm{T}_{3}$ & $16(34 \%)$ & $21(44.7 \%)$ \\
\hline $\mathrm{T}_{4}$ & 9 (19.1\%) & $17(36.2 \%)$ \\
\hline \multicolumn{3}{|l|}{ Nodal involvement } \\
\hline $\mathrm{N}_{0}$ & 34 (72.3\%) & 18 (38.3\%) \\
\hline $\mathrm{N}_{1}$ & $13(27.7 \%)$ & $29(61.7 \%)$ \\
\hline \multicolumn{3}{|l|}{ Distant metastasis } \\
\hline $\mathrm{M}_{0}$ & 44 (93.6\%) & 39 (83\%) \\
\hline $\mathrm{M}_{1}$ & $3.0(6.4 \%)$ & $8.0(17 \%)$ \\
\hline
\end{tabular}

There was no association of high CAA density in tumor with such clinicopathological characteristics as gender, age, tumor location, grade, meanwhile it was determined the higher probability of availability of high density of CAA in tumors among patients at stages III-IV as compared to I-II (odds ratio 5.54, $\chi^{2}=$ 15.2, 95\% CI 27.9-1.71, $p<0.01$ ), in tumor of patients with category $\mathrm{T}_{3-4}$ as compared to $\mathrm{T}_{1-2}$ (odds ratio 4.26, $\chi^{2}=8.89$, 95\% CI 24.9-1.11, $p<0.01$ ), with $\mathrm{N}_{1}$ as compared with $\mathrm{N}_{0}$ (odds ratio $3.56, \chi^{2}=$ 8.68, 95\% CI 15.9-1.91, $p<0.01)$.

Overall, 39.5\%, 46.4\%, 89.5\% of patients with BMI $<25,25<\mathrm{BMI}<30$, BMI $>30$, respectively, had high CAA $(>\mathrm{M})$ in tumors. The probability of availability of high density of CAA in tumor of patients with BMI $>30$ is increased by a factor of 11 (OR 11.01, $\chi^{2}=12.9,95 \%$ CI 28.933-5.749, $p<0.01$ ) as compared with BMI $<25$ and by a factor of almost 9 (OR 8.84, $\chi^{2}=13.47,95 \%$ CI 16.777-4.665, $p<$ $0.01)$ as compared with BMI $<30$.

Data obtained demonstrate that adipocytes are as a major component of the microenvironment of gastric cancer, especially in obesity.

\subsection{CD8- and CD45RO-T Positive Cells in Tumors of} Patients according to BMI

Overall, $80 \%$ and $87.1 \%$ of patients had tumors with $\mathrm{CD}^{+}$- and $\mathrm{CD}^{-} 5 \mathrm{RO}^{+}$-T lymphocytes during 
follow-up, respectively. There was no association between presence of CD8- or CD45RO-positive T cells in tumor and clinicopathological characteristics. The median number of $\mathrm{CD}^{+}$- and $\mathrm{CD}^{2} 5 \mathrm{RO}^{+}$-T cells was $34.4 \%$ (range 5.0-78.2) and 36.4\% (range 5.0-92.2), respectively, and classified all cases into high- or low-density groups for each marker, i.e., the number of $\mathrm{CD}^{+}$- and $\mathrm{CD} 45 \mathrm{RO}^{+}-\mathrm{T}$ cells.

When tumors of patients with BMI $<25,25<$ BMI $<30$, BMI $>30$ were characterized by high density of CAA, high number of $\mathrm{CD}^{+}-\mathrm{T}$ cells was detected in $58.8 .1 \%, 37.5 \%$ and $16.6 \%$ and $\mathrm{CD}_{45 \mathrm{RO}^{+}}-\mathrm{T}$ cells in $57.8 \%, 41.4 \%$ and $30.7 \%$ of cases, respectively.

The mean number of $\mathrm{CD}^{+}$- and $\mathrm{CD}^{-} 4 \mathrm{RO}^{+}-\mathrm{T}$ cells in tumors with high density of CAA was $57.8 \pm 1.7 \%$, $37.3 \pm 4.2 \%$ and $25.7 \pm 1.9 \%$ as well as $41.1 \pm 2.3 \%$ $35.4 \pm 3.4 \%, 26.5 \pm 2.1 \%$ in patients with BMI $<25$, $25<\mathrm{BMI}<30$, BMI $>30$, respectively. Mean number of $\mathrm{CD}^{+}-\mathrm{T}$ cells and $\mathrm{CD}^{2} 5 \mathrm{RO}^{+}-\mathrm{T}$ cells decreased significantly in patients with BMI $>25$ as compared with patients with BMI $<25$ (OR = 6.39, $\chi^{2}=5.86$, 95\% CI 13.735-2.97, $p<0.05$ and OR $=4.08, \chi^{2}=$ 4.309, 95\% CI 7.89-2.10, $p<0.05$, respectively), and mean number of $\mathrm{CD} 8^{+}-\mathrm{T}$ cells in patients with $\mathrm{BMI}>$ $30\left(\mathrm{OR}=4.51, \chi^{2}=4.38,95 \%\right.$ CI 10.486-1.916, $p<$ 0.05 ) as compared with patients with $\mathrm{BMI}<30$.

When tumors were characterized by low CAA it makes no difference between groups of patients with $\mathrm{BMI}<25,25<\mathrm{BMI}<30$, BMI $>30$ concerning the availability of high number of $\mathrm{CD} 8^{+}-\mathrm{T}$ cells as well as $\mathrm{CD}^{2} 5 \mathrm{RO}^{+}-\mathrm{T}$ cells in tumors and the mean number of $\mathrm{CD}^{+}$- and $\mathrm{CD}^{-} 5 \mathrm{RO}^{+}-\mathrm{T}$ cells in tumors was the following: $43.7 \pm 3.3 \%, 42.2 \pm 1.9 \%$ and $39.3 \pm 2.8 \%$, and $45.9 \pm 2.1 \%, 46.3 \pm 2.2 \%$ and $35.7 \pm 3.9 \%$, respectively.

Taking into account ground of our study immunological questions to explain the peculiarities of metabolic symbiosis between TILs and adipocytes would not be discussed because they need special examination.

\subsection{Hypoxia in Tumors according to BMI}

Hypoxia occurs in many pathological conditions, including chronic inflammation and tumors, and is considered to be an inhibitor of $\mathrm{T}$ cell function. We investigated how hypoxic conditions affect density of TILs infiltration in patients according to BMI.

Overall, $35.6 \%$ and $64.4 \%$ of patients had hypoxic tumors and satisfactory oxygenated, respectively. There was no obtained correlation between density of CAA and the level of hypoxia in tumors but it was detected that low density of $\mathrm{CD}^{+}$- and $\mathrm{CD} 45 \mathrm{RO}^{+}-\mathrm{T}$ cells was found in $34.4 \%$ and $46.6 \%$ of patients with hypoxic tumors characterized by high density of CAA and $22.2 \%$ and $16.7 \%$ of patients with low density of CAA, respectively, which demonstrates the predominant role of CAA in this process. But in tumors characterized by high density of CAA slight infiltrating of TILs may be observed under satisfactory oxygenation also: in patients with BMI > 30 the probability of the presence of low density of $\mathrm{CD}^{+}-\mathrm{T}$ cells in tumors is increased by a factor of 6.67 (OR 6.67, $\chi^{2}=5.1$, 95\% CI 15.64-2.835, $p<0.05$ ), but for CD45RO ${ }^{+}$-T cells it is not statistically significant $\left(\chi^{2}=3.05, p>0.05\right)$. For CD45RO ${ }^{+}-\mathrm{T}$ cells it is true for all patients independently of BMI (OR 5.758, $\chi^{2}=$ 4.57, 95\% CI 12.988-2.55, $p<0.05$ ) only. Presented data demonstrate that in tumors characterized by high density of CAA slight infiltrating of $\mathrm{CD}^{+}-\mathrm{T}$ cells may be observed under satisfactory oxygenation also.

It is known that there are specific changes associated with obese adipose tissue, giving rise to altered conditions within the tumor microenvironment. Excess adiposity is associated with an increase in free fatty acids in the environment, and numerous studies have looked at fatty acid metabolism as a potential mechanism to link obesity to cancer. Zhang et al. [26] have shown that hypoxia induces $\mathrm{CD}^{+}$TILs to enhance fatty acid catabolism to preserve functions. It is possible to assume abundant availability of lipids from adipocytes in the tumor microenvironment, but 
not only hypoxia is likely to play a role in the obesity-associated process of metabolic reprogramming of $\mathrm{T}$ cells. Although our understanding of how adipocytes influence tumor progression has grown significantly, the mechanisms of adipocytes impact on TILs infiltration under obesity are not well-understood and need special examination.

Knowledge of pathophysiological mechanisms underlying the association between obesity and tumor TIL infiltration may be important for the development of preventive and therapeutic strategies for cancer [1].

\subsection{CD8- and CD45RO-Positive Cells in BM in} Patients according to BMI

Overall, $80.3 \%$ and $81.2 \%$ of patients had $\mathrm{CD}^{+}$and $\mathrm{CD}_{45 \mathrm{RO}^{+}-\mathrm{T}}$ cells in BM. There were no associations between presence of CD8- or CD45RO-positive $\mathrm{T}$ cells in $\mathrm{BM}$ and clinicopathological characteristics. It was not established a link between presence of TILs in BM: $82.4 \%, 87.5 \%$ and $92.3 \%$ and $83.6 \%, 65.6 \%$ and 93.2\% of patients with BMI $<25$, $25<$ BMI $<30$, BMI $>30$, respectively had $\mathrm{CD}^{+}-$and $\mathrm{CD}^{-} 5 \mathrm{RO}^{+}-\mathrm{T}$ cells in BM.

$\mathrm{CD}^{+}$- and $\mathrm{CD} 45 \mathrm{RO}^{+}-\mathrm{T}$ cells in BM were detected in $81.2 \%, 80.5 \%$ and $79.1 \%, 87.0 \%$ when tumors were characterized by low and high density of CAA in tumor, respectively.

CD8-positive $\mathrm{T}$ cells were found in $77.8 \%$ of patients with tumors characterized by severe and moderate hypoxia (PME/Pi < 1.4) and in $90.90 \%$ of patients with tumor characterized by mild and weak hypoxia (PME/Pi > 1.4), and for CD45RO-positive $\mathrm{T}$ cells: $93.8 \%$ and $94.4 \%$ of patients, respectively. The association between presence of CD8- and CD45RO-positive T cells in BM and hypoxia level in primary tumors characterized by high density of CAA was not detected.

Obtained results have shown that tumor hypoxia does not associate with the presence of CD8 and CD45RO $\mathrm{T}$ cells in $\mathrm{BM}$ and, probably, does not influence their activity.

\subsection{Overall Survival of Patients with Low Density of} TILs according to BMI

The association between overall survival of the patients in accordance with number of TILs and density of CAA in tumor has been shown. Survival of patients was estimated and evaluated by Kaplan-Meier curves. Low CAAs have been in $11.6 \%$ of patients with BMI $>30$ that prevented us to provide statistical analysis.

Taking into account that majority of patients with BMI > 30 (88.4\%) have tumors with high density of CAA and slight infiltrating of TILs (79.2\%) we compared survival of patients with BMI $>30$ with survival of patients with BMI $<25$ who have tumors also with high density of CAA and slight infiltrating of TILs.

Moreover it was compared the overall survival of patients with BMI $<25$ with slight number of TILs in tumor who have both high and low density of CAA in tumors.

Patients both having the slight number of $\mathrm{CD}^{+}$and $\mathrm{CD} 45 \mathrm{RO}^{+}-\mathrm{T}$ cells and high density of CAA in tumors, who had BMI > 30 demonstrated better survival than patients with BMI $<25$ (log rank test: $p$ $=0.04818$, and 0.04669, respectively) with medians follow-up time 24.7 (range, 7.1-32.38) and 17.9 (range, 8.1-26.8) months from diagnosis, respectively whereas patients with BMI $<25$ having slight number of TILs and low density of CAA in tumors have shown much longer overall survival time than patients with high density of CAA (log rank test: $p=0.02242$ and $p=0.00964$ ) with median follow-up time 25.1 (range, 9.3-48.2) months from diagnosis. It may be supposed that when there is slight number of TILs in tumor, high density of CAA plays a key role in the overall survival of patients.

It may be suggested that high density of CAA in tumor plays a key role in the overall survival of patients with BMI $>30$, but it is not true for the patients with $\mathrm{BMI}<25$ (Figs. 1 and 2). 


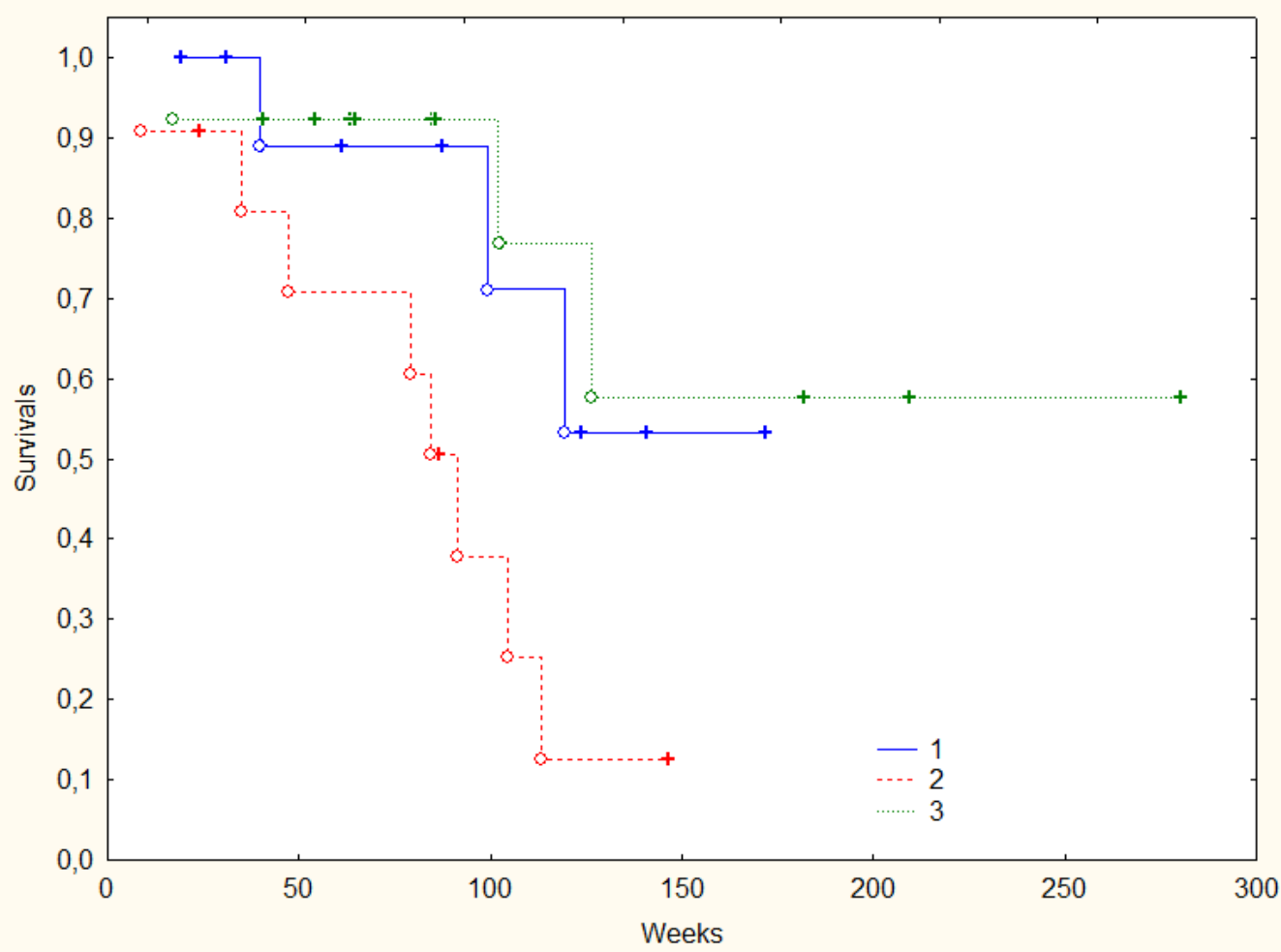

Fig. 1 Kaplan-Meier OS curves for gastric cancer patients according to low density CD8 ${ }^{+}$T cells in tumor tissue: (1) patients with BMI > 30 and high density of CAA in tumor; (2) patients with BMI < 25 and high density of CAA in tumor; (3) patients with BMI $<25$ and low density of CAA in tumor; log-rank test $p=0.04818(1-2), p=0.02242(3-2)$.

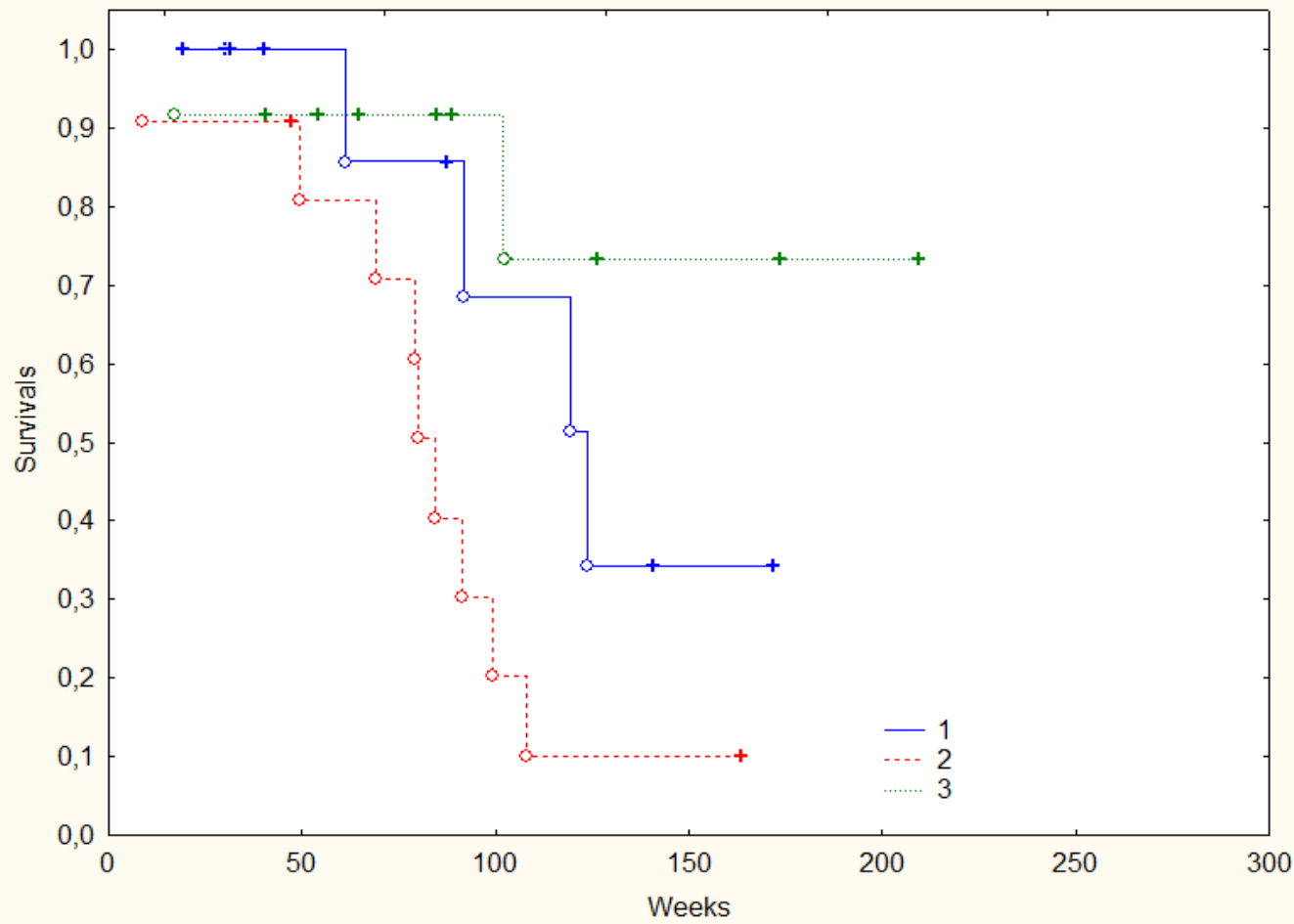

Fig. 2 Kaplan-Meier OS curves for gastric cancer patients according to low density $\mathrm{CD}^{2} \mathrm{RO}^{+}$-T cells in tumor tissue: (1) patients with BMI > 30 and high density of CAA in tumor; (2) patients with BMI < 25 and high density of CAA in tumor; (3) patients with BMI < 25 and low density of CAA in tumor; log-rank test: $p=0.04669(1-2), p=0.00964(3-2)$. 
It was not seen any associations between presence of TILs in BM and overall survival of patients according to BMI.

\section{Conclusions}

In this study we found that elevated BMI has been consistently associated with high density of CAA in tumors. The density of TIL infiltrating in samples of gastric cancer was significantly associated with BMI patients having high density of CAA but not with hypoxia level in tumor tissue. Patients with BMI > 30 having high density of CAA in tumors with slight infiltrating of TILs demonstrated better overall survival than the same patients with BMI $<25$ whereas the same patients with BMI $<25$ but with low density of CAA in tumors have shown much longer overall survival time.

Presence of TILs in BM has not been associated with BMI. There was no observed influence of hypoxia in primary tumor in this process. Presence of $\mathrm{CD}^{+}$- and $\mathrm{CD}^{-} 5 \mathrm{RO}^{+}-\mathrm{T}$ cells in BM was not affected by the high density of CAA in primary tumor. There were no seen associations between presence of TILs in BM and overall survival of patients according to BMI also.

Therefore further clarification of the associations between obesity and cancers may be taking into account to improve prognosis and may help to pave the way for more effective treatments of patients having overweight.

\section{Acknowledgment}

This research was supported by the National Academy of Sciences of Ukraine (Grants No. 2.2.5.390 and No. 0114U004392).

\section{Conflict of Interests}

The authors report no conflict of interests. The authors alone are responsible for the content and writing of the paper.

\section{References}

[1] Van Kruijsdijk, R. C. M., Van der Wall, E., and Visseren, F. L. J. 2009. "Obesity and Cancer: Role of Dysfunctional Adipose Tissue.” Cancer Epidemiol Biomarkers Prev 18: 2569-78.

[2] Calle, E. E., and Overveit, K. R. 2004. “Obesity and Cancer: Epidemiological Evidence and Proposed Mechanisms.” Nature Rev Cancer 4: 579-91.

[3] Park, Y., Peterson, L. L., and Colditz, G. A. 2017. "The Plausibility of Obesity Paradox in Cancer-Point.” Compr Physiol 1:237-82.

[4] Kyrgiou, M., Kalliala, I., Markozannes, G., et al. 2017. "Adiposity and Cancer at Major Anatomical Sites: Umbrella Review of the Literature.” BMJ 356: j477. doi: 10.1136/bmj.j477.

[5] Cozzo, A. J., Fuller, A. M., and Makowski, L. 2017. "Contribution of Adipose Tissue to Development of Cancer.” Dental Press J Orthod 3: 21-5.

[6] Vaupel, P., Mayer, A., and Höckel, M. 2004. "Tumor Hypoxia and Malignat Progression." Methods in Enzymology 381: 335-54.

[7] Nieman, K. M., Romero, I. L., Van Houten, B., and Lengyel, E. 2013. "Adipose Tissue and Adipocytes Support Tumorigenesis and Metastasis.” Biochim Biophys Acta 10: 1533-41.

[8] Clark, C. E., Hingorani, S. R., Mick, R., et al. 2007. "Dynamics of the Immune Reaction to Pancreatic Cancer from Inception to Invasion.” Cancer Res 67: 9518-27.

[9] Enomoto, K., Sho, M., Wakatsuki, K., et al. 2012. "Prognostic Importance of Tumor-Infiltrating Memory T Cells in Oesophageal Squamous Cell Carcinoma.” Clin Exp Immunol 168: 186-91.

[10] Mahmoud, S. M. A., Paish, E. C., Powe, D. G., et al. 2011. "Tunor-Infiltrating $\mathrm{CD}^{+}$Lymphocytes Predict Clinical Outcome in Breast Cancer.” J Clin Oncol 29: 1949-55.

[11] Loi1, S., Michiels, S., Salgado, R., et al. 2014. "Tumor Infiltrating Lymphocytes Are Prognostic in Triple Negative Breast Cancer and Predictive for Trastuzumab Benefit in Early Breast Cancer: Results from the FinHER Trial.” Ann Oncol 25: 1544-50. doi: 10.1093/annonc/mdu112.

[12] Balermpas, P., Michel, Y., Wagenblast, J., et al. 2014. “Tumor-Infiltrating Lymphocyte Predict Response to Definitive Chemoradiotherapy in Head and Neck Cancer.” Brit J Cancer 110: 501-9.

[13] Pages, F., Kirilovsky, A., Mlecnik, B., et al. 2009. “In Situ Cytotoxic and Memory T Cells Predict Outcome in Patients with Early-Stage Colorectal Cancer.” J Clin Oncol 27: 5944-51. 


\section{Patients with Gastric Cancer and Overweight}

[14] Bachmayr-Heyda, A., Aust, S., Heinze, G., et al. 2013. "Prognostic Impact of Tumor Infiltrating $\mathrm{CD}^{+} \mathrm{T}$ Cells in Association with Cell Proliferation in Ovarian Cancer Patients-A Study of OVCAD Consortium." BMC Cancer 13: 422-9.

[15] Wakatsuki, K., Sho, M., Yamato, I., et al. 2013. "Clinical Impact of Tumor-Infiltrating CD45RO ${ }^{+}$ Memory T Cells on Human Gastric Cancer.” Oncol Reports 29: 1756-62.

[16] Osinsky, S., Kovelskaya, A., Bubnovskaya, L., et al. 2015. "CD8 and CD45RO T Lymphocytes in Bone Marrow of Gastric Cancer Patients: Correlation with Disseminated Tumor Cells and Disease Outcome.” Exp Oncol 37: 48-52.

[17] Mei, Z., Liu, Y., Liu, C., et al. 2014. “Tumor-Infiltrating Inflammation and Prognosis in Colorectal Cancer: Systemic Review and Meta-Analysis.” Brit J Cancer 110: 1595-605.

[18] Helal, T. E., Ibrahim, E. A., and Alloub, A. I. 2013. "Immunohistochemical Analysis of Tumor-Infiltrating Lymphocytes in Breast Carcinoma: Relation to Prognostic Variables.” Indian J Pathol Microbiol. 56 (2): 89-93. doi: 10.4103/0377-4929.118676.

[19] Hotta, K., Sho, M., Fujimoto, K., et al. 2011. "Prognostic Significance of $\mathrm{CD}_{4} 5^{+}$Memory T Cells in Renal Cell Carcinoma.” Br J Cancer 105: 1191-6.

[20] Pagès, F., Galon, J., Dieu-Nosjean, M. C., et al. 2010. "Immune Infiltration in Human Tumors: A Prognostic Factor that Should Not Be Ignored.” Oncogene 29:
1093-102.

[21] Gooden, M. J., De Bock, G. H., Leffers, N., et al. 2011. "The Prognostic Influence of Tumour-Infiltrating Lymphocytes in Cancer: A Systematic Review with Meta-Analysis.” Br J Cancer 105: 93-103.

[22] International Union against Cancer. 2002. TNM Classification of Malignant Tumors, 6th ed., edited by Sobin, L. H., and Wittekind, C., New York, USA: Wiley-Liss.

[23] Fenoglio-Preiser, C., Carneiro, F., Correa, P. et al. 2000. "Gastric Carcinoma." in World Health Organization Classification of Tumors: Tumours of the Stomach, edited by Hamilton, S. R., and Aaltonen, L. A., Vol. 3, Chapter 3, pp. 39-52, Lyon, France: IARC Press.

[24] Vaupel, P., Okunieff, P., Kallinowski, F., and Neuringer, L. J. 1989. "Correlation between ${ }^{31} \mathrm{P}-\mathrm{NMR}$ Spectroscopy and Tissue $\mathrm{O}_{2}$ Tention Measurements in a Murine Fibrocarcoma.” Radiation Res 120: 477-93.

[25] Bubnovskaya, L. M., Kovelskaya, A. V., Boldeskul, I. E., et al. 2011. "Assesment of Tumor Hypoxia and Hypoxia-Related Metabolites by NMR Spectroscopy and Prognostic Relevance.” In Tumor Hypoxia in the Clinical Setting, edited by Osinsky, S., Friess, H., and Vaupel, P., Kiev: Akademperiodica, 203-19.

[26] Zhang, Y., Kurupati, R., Liu, L., et al. 2017. "Enhancing $\mathrm{CD}^{+} \mathrm{T}$ Cell Fatty Acid Catabolism within a Metabolically Challenging Tumor Microenvironment Increases the Efficacy of Melanoma Immunotherapy." Cancer Cell 32: 377-91. 